\title{
Évolution récente des débits dans la basse vallée de l’Ouémé, Sud-Benin
}

\section{Fêmi COCKER ${ }^{1}$, Jean-Bosco K. VODOUNOU ${ }^{2}$, Jacob A. YABI ${ }^{3}$}

${ }^{1}$ Ecole Doctorale des Sciences Agronomiques et de 1'Eau, femicocker@gmail.com

${ }^{2}$ Laboratoire des Géosciences de l'Environnement et de la Cartographie,vjeanbosco@gmail.com

${ }^{3}$ Laboratoire d'Analyses et de Recherches sur les Dynamiques Économiques et Sociales, jacob.yabi@,fa-up.bj

\section{INFOS SUR L'A R T I C L E}

Historique de l'article:

Reçu le : 31 juillet 2019

Reçu en format revisé le : 24 novembre 2019

Accepté le : 26 novembre 2019

Mots-Clés : Évolution des débits/

fluctuations pluviométriques / bilan

hydrologique / basse vallée de l'Ouémé

Keywords: Changes in flows/pluviometric fluctuations / hydrological balance / lower Oueme valley

\begin{abstract}
RES U M E / A B S T R A C T
Résumé: L'objectif de cette étude est de caractériser l'évolution des débits dans la basse vallée de l'Ouémé au sud-Bénin. Les données utilisées, sont les débits, les pluies et l'évapotranspiration potentielle, collectées sur une série de 30 ans. Les outilstels que ArcGIS, Xlstat et Khronostatont servi à leur traitement. Ces informations ont aidé à caractériser l'évolution des débits dans la zone d'étude. Les résultats obtenus montrent une inégale répartition des débits.L'étude a identifié deux phases mettant en exergue deux sous périodes, 1987-2007 et 2008-2016. La première est déficitaire avec une moyenne du débit égale à $194 \mathrm{~m}^{3} \mathrm{~s}^{-1}$, tandis que la seconde est excédentaire et caractérisée par une moyenne de $221 \mathrm{~m}^{3} \mathrm{~s}^{-1}$. Cette variation à la hausse est le signe d'une amélioration des conditions du système hydrologique et d'une disponibilité en eau dans le bassin de l'Ouémé. Ces résultats serviront à améliorer les performances agricoles et l'éclosion d'autres filières de développement pour impacter l'ensemble de la vallée de l’Ouémé, deuxième vallée la plus riche du monde après le Nil.
\end{abstract}

Abstract: The objective of this study is to characterize the evolution of flows in the lower Ouemé valley in south-Benin. The data used are flows, rains and potential evapotranspiration collected over a series of 30 years. Tools such as ArcGIS, Xlstat and Khronostat have been used to treat them. This information helped to characterize the evolution of flows in the study area. The results show an uneven distribution of flows. The study identified two phases highlighting two subperiods, 1987-2007 and 2008-2016. The first is in deficit with an average flow of $194 \mathrm{~m}^{3} \mathrm{~s}^{-1}$, while the second is surplus and characterized by an average of $221 \mathrm{~m}^{3} \mathrm{~s}^{-1}$. This upward trend is a sign of improved hydrological system conditions and water availability in the Oueme watershed. These results will be used to improve agricultural performance and the emergence of other development streams to impact the entire Oueme valley, the second richest valley in the world after the Nile.

\section{INTRODUCTION}

La variation des débits est en lien avec les quantités de pluies enregistrées. C'est l'alerte que donnait l'Autorité du Bassin du fleuve Niger, neuf États, (le Bénin, le Burkina Faso, le Cameroun, la Côte d'Ivoire, la Guinée, le Mali, le Niger, le Nigeria et le Tchad)en s'inquiétant depuis septembre 2017 de la montée des eaux suite à la crue favorisée par les fortes pluies qui se sont abattues en juin 2017 sur le Mali et le Niger, redoutant ainsi des inondations pires que celles de 2012 qui avaient fait des dizaines de morts et près de 500000 sinistrés au Niger AFP, 2017. En effet, les variabilités hydrométriques ont un impact direct sur les ressources en eau, puisqu'en partie, le renouvellement de la ressource et la recharge de la nappe en dépendent. Cet impact est particulièrement important dans les pays en développement où les techniques de mobilisation de ressources en eau ne sont pas encore diversifiées ou parfois restent à l'étape embryonnaire. En Afrique, et au Bénin en particulier, les grands fleuves comme le fleuve Ouémé, subissent une forte pression démographique liée à leur situation géographique Mama, 2010.Plusieurs études ont été réalisées dans le bassin de l'Ouémé selon Le Lay, 2002 ; Peugeot et al, 2011 ; Zannou, 2011 ; Akognongbé, 2014 et Abdoulaye, 2015 sur des thématiques similaires. La présente étude vise à caractériser la variabilité hydrométrique dans cinq communes de la basse vallée de l'Ouéméque sont Bonou, Adjohoun, Dangbo, Aguégués et Sô-Ava (fig.1) 


\section{PRESENTATION DU MILIEU D'ETUDE}

La figure 1 (Fig. 1) présente la situation géographique du milieu d'étude. Il s'agit d'une plaine d'inondation localisée au sud-est du Bénin et est comprise entre $2^{\circ} 21^{\prime} 2^{\prime \prime}$ et $2^{\circ} 36^{\prime} 5^{\prime \prime}$ de longitude est et entre $6^{\circ} 24^{\prime} 5^{\prime \prime}$ et $6^{\circ} 58^{\prime} 1 "$ de latitude nord. Les communes de Bonou, Adjohoun, Dangbo, Aguégués et Sô-Ava dans la basse vallée de l'Ouémé couvrent une superficie de $1236 \mathrm{~km}^{2}$. Le climat est de type subéquatorial à quatre saisons d'inégale répartition, deux saisons pluvieuses et deux saisons sèches. La densité de population est en moyenne de 215 habitants au km Codjia, 2009.

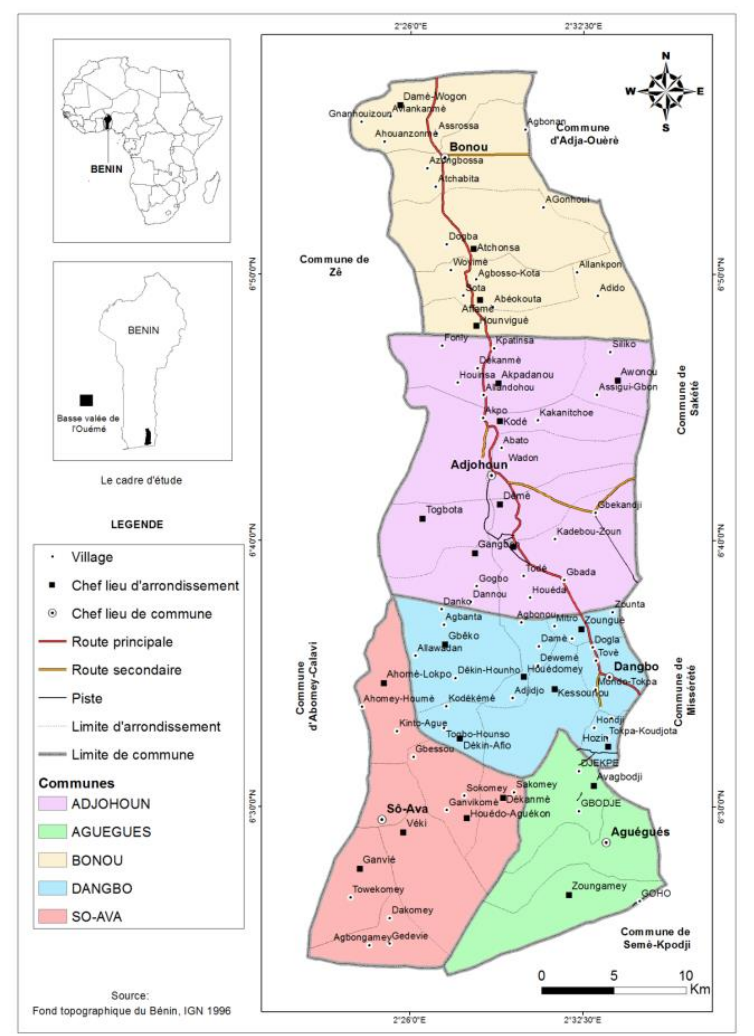

Fig1: Présentation du milieu d'étude

\section{APPROCHE METHODOLOGIQUE}

Afin de mettre en évidence l'évolution des débits dans la basse vallée de l'Ouémé, des données hydrométriques, pluviométriques et l'évapotranspiration ont été collectées et analysées sur une série de 30 ans. Les données hydrométriques ont été recueillies à la Direction Générale de l'Eau (DGEau) et concernent les chroniques hydrologiques de la station de Bonou. Les données pluviométriques et l'évapotranspiration ont été obtenues à l'Agence pour la Sécurité de la Navigation Aérienne (ASECNA) et concernent les stations de Cotonou, Bohicon, Savè, Parakou, Natitingou, Kandi, Porto-Novo, Adjohoun et Bonou.La caractérisation de l'évolution des débits dans la basse vallée de l'Ouémé a été faite à travers la détection de rupture (test de Pettitt), le calcul des anomalies centrées réduites, les déficits, les paramètres de tendance centrale et de dispersion. L'utilisation du coefficient de corrélation linéaire de Bravais-Pearson cité par Vissin, 2007 a permis de détecter tour à tour la présence d'une relation linéaire entre les lames d'eau écoulées et les termes du bilan. Ces données ont été complétées par des observations de terrain.

\section{RESULTATS ET DISCUSSION}

\section{IV.1. Variabilité mensuelle des débits dans la basse vallée de l'Ouémé}

L'analyse de la répartition mensuelle des débits enregistrés a permis de caractériser le régime hydrologique moyen du milieu d'étude (Fig. 2).

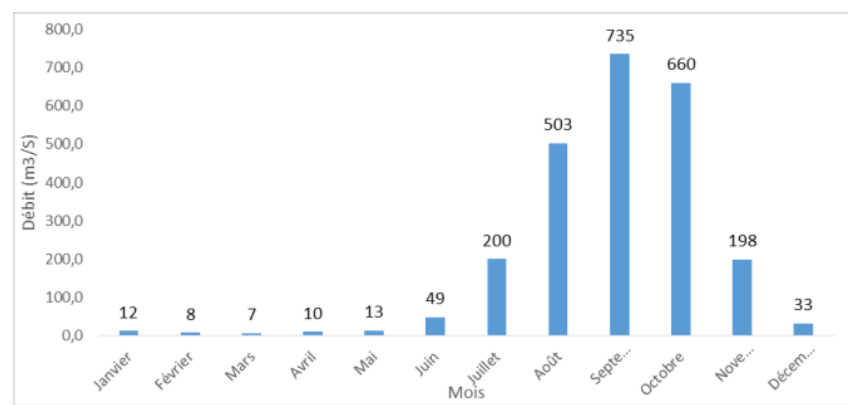

Fig2: Évolution des débits mensuels sur la série étudiée (1987-2016)

De l'analyse de la (Fig. 2), il ressort que les mois d'août, septembre et octobre enregistrent les débits les plus élevés du fleuve dans la basse vallée de l'Ouémé ( $>$ $\left.500 \mathrm{~m}^{3} \mathrm{~s}^{-1}\right)$. Le pic est atteint en septembre avec une valeur maximale de $735 \mathrm{~m}^{3} \mathrm{~s}^{-1}$. Cette période (août, septembre et octobre) à elle seule, représente environ 80 $\%$ du débit annuel. Ainsi, les débits varient de façon considérable au cours d'une même année.

Ces résultats sont similaires à ceux de Laleye et al, 2004, qui situent l'inondation dans le bassin de fin août à mi-octobre de façon générale, tout en nuançant qu'elle peut survenir dès juillet pour se terminer en début novembre comme les observations de terrain en 2017 dans le milieu d'étude (Fig. 3). De même, Amoussou, 2015 dans son étude sur l'hydrométéorologie des crues dans le bassin-versant du Mono, constate que les crues maximales annuelles sont enregistrées au cours de l'été. Les plus faibles débits quant à eux, sont enregistrés durant la période sèche de chaque année (décembre à avril, voire mai). De ce fait, la décrue est amorcée en novembre et atteint son niveau critique en février et mars. Ce qui confirme qu'en année très sèche, il peut ne pas se produire de crue du tout Welcomme,1971; Nonfon,1988; Laleye, et al 2004. 


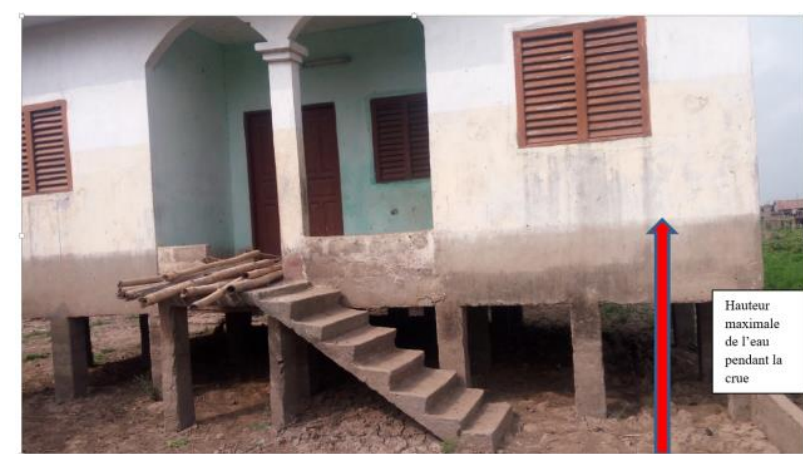

Fig 3 : Niveau de l'eau atteint à Dogodo-Houinta dans les Aguégués lors de la crue de septembre 2017 Cliché : F. COCKER, 15/01/2018

Le retrait des eaux observé sur la (Fig. 3), au cours du mois de janvier, indique en même temps le niveau maximum de l'eau pendant la période des crues, (août, septembre et octobre). La flèche en rouge montre le niveau maximum de la montée des eaux. Ce calendrier des crues est similaire à celui obtenu par Moniod, 1973 qui a démontré que le régime hydrologique de 1'Ouémé est caractérisé par un débit minimal au mois de mars et un débit maximal pendant la période des hautes eaux au mois de septembre, mais la période de basses eaux s'étend de janvier à mai inclus. La crue arrive en juin et le débit croît jusqu'en septembre ; il se maintient au voisinage du maximum pendant le mois d'octobre.

Le nombre important de plans d'eau dans la vallée constitue également, un élément fondamental dans la manifestation de ces inondations en ce sens que l'eau qui s'y écoule sature les sols et diminue leur capacité d'infiltration. Cependant, les travaux de Cocker et al, 2018, indiquent que les rivières dans ces mois sont alimentées en surplus d'eau et favorisent l'alimentation des réservoirs souterrains des sous bassins versants. L'évolution au fil des années sera caractérisée par la variation interannuelle des débits dans le milieu.

\section{IV.2. Variation interannuelle des débits dans la basse vallée de l'Ouémé}

La (Fig.4) présente les débits annuels exprimés par la variable centrée réduite.

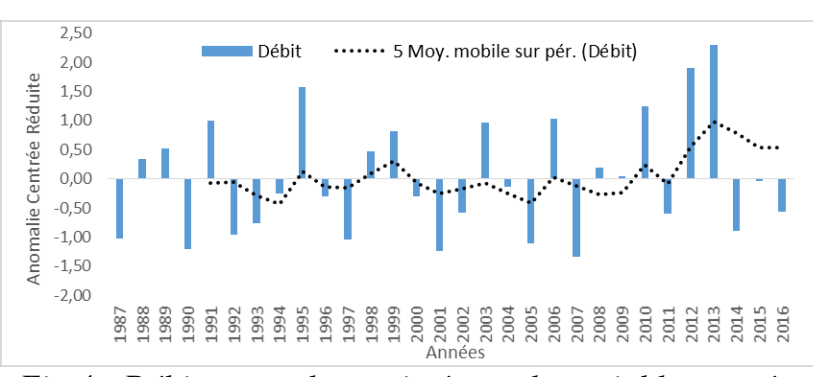

Fig 4 : Débits annuels exprimés par la variable centrée réduite

L'analyse des anomalies centrées réduites permet de dire qu'une variation importante des débits moyens annuels est observée sur toute la série. Dans cette période, ce sont les années 1995, 2010, 2012 et 2013 qui ont les débits les plus élevés. Par contre, 56\% des années de la série chronologique présentent un déficit. Ainsi, les années de grands déficits d'écoulement enregistrés dans la basse vallée sont 1987, 1990, 1997, 2001, 2005 et 2007. Les déficits de ces périodes correspondent aux déficits pluviométriques enregistrés dans le milieu d'étude entre1990 et 2006par Cocker et al, 2018. Ces résultats sont comparables à l'étude de Amoussou, 2015 qui est parvenu à une instable répartition des débits dans le bassin-versant du Mono.

Le test de Pettitt appliqué aux séries hydrométriques a permis de détecter une rupture de stationnarité significative au seuil de $95 \%$ en 2007 (Fig. 5).

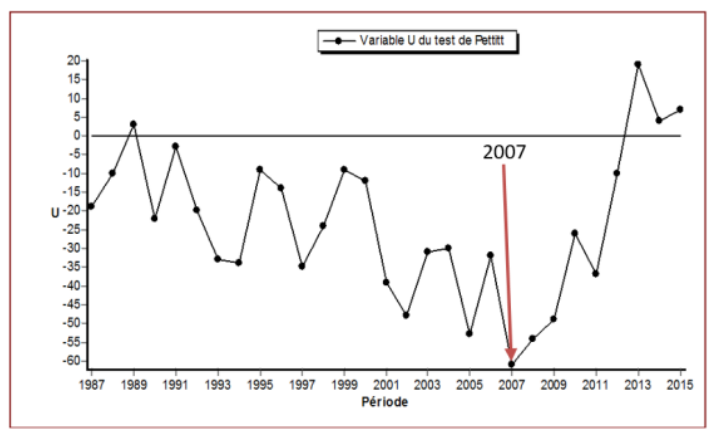

Fig 5 : Rupture de stationnarité dans les débits annuels

A partir de ce test, deux sous périodes sont identifiées dans la série chronologique. Il s'agit des périodes 19872007 et 2008-2016. La période 1987-2007 est déficitaire avec une moyenne du débit égale à $194 \mathrm{~m}^{3} \mathrm{~s}^{-1}$, tandis que la période 2008-2016 est excédentaire et caractérisée par une moyenne de $221 \mathrm{~m}^{3} \mathrm{~s}^{-1}$.

\section{IV.3. Répartition intra-saisonnière comparée des écoulements des sous-périodes 1987-2007 et 2008-2016}

La comparaison du régime hydrologique des deux périodes dans la basse vallée de l'Ouémé est présentée par la (fig. 6)

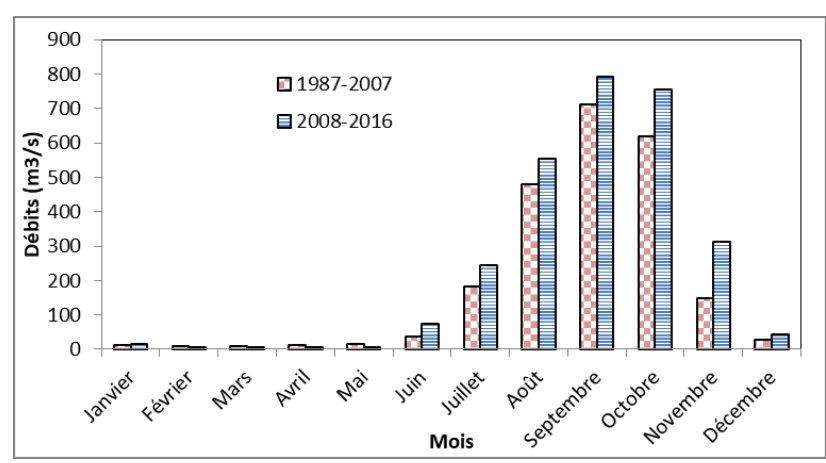

Fig 6 : Variation saisonnière des débits sur les souspériodes 1987-2007 et 2008-2016.

Les mois d'octobre et novembre ont subi les plus fortes augmentations d'écoulement entre les deux sous périodes. Le retour à des situations pluviométriques plus favorables à partir de la deuxième moitié de la décennie 2000, a donc amélioré la situation des écoulements et présage d'une disponibilité en eau dans la basse vallée de 
l'Ouémé. Toutefois, on observe un écart conséquent $\left(24.7 \mathrm{~m}^{3} \cdot \mathrm{s}^{-1}\right)$ entre la première période et la deuxième.

\section{IV.4. Bilan hydrologique dans la basse vallée de l'Ouémé}

La (Fig. 7), présente la variabilité interannuelle des termes du bilan hydrologique dans la basse vallée du fleuve Ouémé.

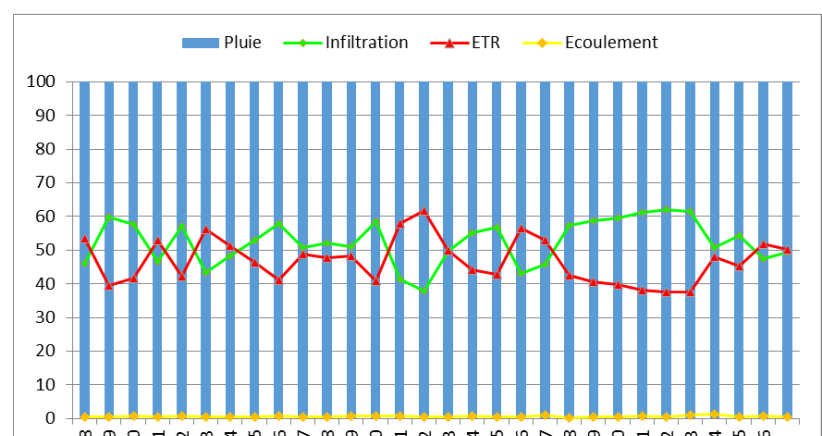

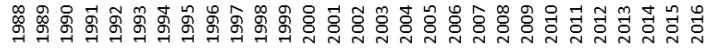

Fig7 : Variabilité interannuelle des termes du bilan hydrologique

L'analyse de la (Fig. 7), révèle que pour une hauteur de pluie de $100 \%$ reçue, on note 36 à $62 \%$ pour l'évaporation, 38 à $62 \%$ pour la recharge et l'écoulement oscille entre 0,2 et $1,2 \%$. Il y a donc d'énormes pertes par évaporation. Aussi, observe-t-on un faible écoulement sur la période d'étude. Cela peut être justifié par la configuration du sol, la géomorphologie ainsi que par le pouvoir évaporatoire du milieu. La Table 1 montre l'évolution comparée des termes du bilan hydrologique en mm dans la basse vallée de l'Ouémé.

Table 1 : Évolution comparée des fluctuations pluviométriques et des autres termes du bilan hydrologique en mm dans le bassin versant de la basse vallée de l'Ouémé

\begin{tabular}{|l|c|c|}
\multicolumn{4}{c|}{} & $\begin{array}{c}\text { Basse vallée de } \\
\text { l'Ouémé }\end{array}$ \\
\hline \multirow{4}{*}{ Pluie } & $1987-2016$ & 98,0 \\
\cline { 2 - 3 } & $1987-2007$ & 97,0 \\
\cline { 2 - 3 } & $2008-2016$ & 100,3 \\
\cline { 2 - 3 } & Écart & $-3,3$ \\
\cline { 2 - 3 } & Déficit & $-3,355$ \\
\hline \multirow{4}{*}{ Évaporation } & $1987-2016$ & 45,3 \\
\cline { 2 - 3 } & $1987-2007$ & 46,4 \\
\cline { 2 - 3 } & $2008-2016$ & 42,9 \\
\cline { 2 - 3 } & Écart & 3,47 \\
\cline { 2 - 3 } & Déficit & 7,49 \\
\hline \multirow{4}{*}{ Écoulement } & $1987-2016$ & 0,6 \\
\cline { 2 - 3 } & $1987-2007$ & 0,6 \\
\cline { 2 - 3 } & $2008-2016$ & 0,7 \\
\cline { 2 - 3 } & Écart & $-0,14$ \\
\cline { 2 - 3 } & Déficit & $-24,66$ \\
\hline \multirow{5}{*}{ Recharge } & $1987-2016$ & 52,1 \\
\cline { 2 - 3 } & $1987-2007$ & 50,1 \\
\cline { 2 - 3 } & $2008-2016$ & 56,7 \\
\cline { 2 - 3 } & Écart & $-6,59$ \\
\cline { 2 - 3 } & Déficit & $-13,16$ \\
\hline
\end{tabular}

Source : Données de terrain, 2018 / ASECNA, 2016
De l'observation de la Table 1, on note un déficit pluviométrique entre les périodes 1987-2007 et 20082016. Ce déficit est de $-3,35 \%$ ce qui confirme la variation à la hausse des hauteurs de pluie dans la basse vallée de l'Ouémé.

Le déficit d'écoulement et de la recharge entre les deux périodes sont importants soit respectivement $24,66 \%$ et- $13,16 \%$ ce qui traduit une hausse de l'écoulement et de la recharge entre les périodes 19872007 et 2008-2016. La hausse de la pluviométrie entre ces deux périodes a des répercussions importantes sur l'écoulement et la recharge. Cette hausse favorise une disponibilité en eau de surface et en eau souterraine dans la basse vallée de l'Ouémé.

Par ailleurs, les flux terrigènes drainés par les eaux contribuent à l'ensablement de la basse vallée. Ce constat permet de comprendre l'exploitation à peine contrôlée du sable sur plusieurs sites le long de la basse vallée avec l'aval des collectivités locales qui se contentent de percevoir des taxes de prélèvement, sans une organisation durable de la filière.

\section{IV.5. Influence des fluctuations pluviométriques sur les termes du bilan}

L'application du test de corrélation de Person au seuil de significativité de $95 \%$ à ces différents paramètres fait ressortir les liaisons existantes entre les termes du bilan hydrologique dans la basse vallée de l'Ouémé (Table 2).

Table 2 : Matrice de corrélation (test de Pearson)

\begin{tabular}{lcccc}
\hline & Écoulement & ETR & Infiltration & Pluie \\
\hline Écoulement & 1 & & & \\
ETR & $-0,260$ & 1 & & \\
Infiltration & 0,51 & $-0,479$ & 1 & \\
Pluie & 0,509 & $-0,280$ & 0,98 & 1 \\
\hline
\end{tabular}

Corrélation significative entre les termes du bilan au seuil de $95 \%$

L'observation de la Table 2 révèle l'existence d'une forte corrélation positive $(r>0,5)$ significative au seuil de $95 \%$ entre les précipitations, l'infiltration et l'écoulement dans la basse vallée de l'Ouémé.

Le coefficient de corrélation entre la pluie et la recharge est le plus élevé $(\mathrm{r}=0,98)$ ce qui témoigne de la forte relation positive entre ces deux facteurs. Aussi, existe-t-il une corrélation positive et significative entre l'écoulement et les précipitations $(\mathrm{r}=0,509)$. Par contre la corrélation entre l'évapotranspiration et l'infiltration est négative $(\mathrm{r}=-0,479)$. 


\section{CONCLUSION}

$\mathrm{Au}$ terme de cette étude, il ressort un caractère irrégulier du débit et une variation de la pluviométrie dans le milieu d'étude. L'application du test de Pettitt à la série hydrométrique a permis de mettre en évidence une rupture de stationnarité au seuil de $95 \%$ en 2007. Ces résultats révèlent également une tendance à la hausse de l'écoulement en lien avec la pluviométrie et l'infiltration. C'est donc une amélioration des conditions hydrologiques favorables à la disponibilité en eau dans la basse vallée de l'Ouémé. Cette disponibilité peut contribuer à l'essor des cultures de contre saison qui peuvent y être repensées et améliorées avec les méthodes modernes d'exploitation. Ce serait ainsi l'amorce de la valorisation de cette riche vallée encore très peu connue.

\section{REFERENCES}

Abdoulaye D., 2015. Dynamique de l'occupation des terres et ses incidences sur l'écoulement dans le bassin de l'Ouémé à l'exutoire de Bétérou (NordBénin) Thèse de doctorat unique de l'Université d'Abomey-Calavi, Bénin, 253p.

AFP, 2017. Niger: 54 morts, près de 200000 sinistrés après des inondations $\mathrm{J}$. Montr. Online: http://www.journaldemontreal.com/2017/09/15/niger54-morts-pres-de-200-000-sinistres-apres-desinondations

Akognongbe S., 2014. Influence de la variabilité climatique et des activités anthropiques sur les eaux de surface dans le bassin de l'Ouémé à Bétérou au Bénin Thèse de doctorat unique de l'Université d'Abomey-Calavi, Bénin, 258p.

Amoussou E., 2015. Analyse hydrométéorologique des crues dans le bassin-versant du Mono en Afrique de l'Ouest avec un modèle conceptuel pluie-débit, FMSH-WP-2015-90. 2014. < halshs-01143318

Amoussou E. et al., 2015. Péjoration climatique et dynamique hydroécologique dans le bassin-versant du fleuve Ouémé à Bonou au Bénin Hydrol. Sci. J. 57, pp.235-44

Cocker F., Vodounou J-B., Zodekon R.et Yabi J., 2018. Disponibilité de la ressource en eau et variabilité climatique dans la basse vallée de l'Ouémé, au sud Bénin (Afrique de l'Ouest) Int. J. Innov. Sci. Res. 38pp. 289-300

Codjia C., 2009. Perceptions, savoirs locaux et stratégies d'adaptations aux changements climatiques des producteurs des communes d'Adjohoun et de Dangbo au Sud-Est Bénin, mémoire d'Ingénieur Agronome, Université d'Abomey-Calavi, 159p

Houndenou C., 1999.Variabilité climatique et maïsiculture en milieu tropical humide : l'exemple du Bénindiagnostic et modélisation Thèse de doctorat de $3^{\circ}$ cycle Dijon, France, 390p.

Laleye P A, Chikou A, Philippapt J-C, Teugels G and Vandewalle P, 2004. Etude de la diversité ichtyologique du bassin du fleuve Ouémé au Bénin (Afrique de l'Ouest) CYBIUM28, pp.329-39

LeLay M., 2002.Caractérisation hydrologique et simulation numérique des écoulements sur le bassin de la haute vallée de l'Ouémé (Bénin) Rapport de $D E A$, Grenoble, France, 56p.

Lubes H, Masson J-M, Raous P and Tapiayu M., 1994. Logiciel de calculs statistiques et d'analyse fréquentielle adapté à l'évaluation du risque en hydrologie ORSTOM, Paris, 140p.

Moniod F., Régime hydrologique de l'Ouémé (Dahomey). Cah. O.R.S.T.O.M., sbr. Hydrol., vol. X, $\mathrm{n}^{\circ} 2$, pp.171-183.

Mama A., 2010. Méthodologie et résultats du diagnostic de l'eutrophisation du lac Nokoué (Bénin) Thèse de Doctorat, Université de Limoges, France, 157p.

Nonfon M., 1988.Données préliminaires sur l'écologie et la production halieutique des "trous à poissons" de la rive gauche de la basse vallée de l'Ouémé (République Populaire du Bénin) Mémoire d'Ingénieur Agronome, FSA, UNB, Bénin, 152p

Pettitt A. N. 1979. A non-parametric approach to the change point problem, Applied Statistics, 28 (2), pp. 16-36.

Peugeot C, Guichard F, Bock O, Bouniol D, Chong D, Boone A., Cappelaer B., Gosset M., Besson L., Lemaitre Y., Seguis L., Zannou A., Galle S. and Redelsperger J-L., 201, Mesoscale water cycle within the West African Monsoon,Atmos.Sci.Let., $\mathrm{n}^{\circ} 12, \mathrm{pp} .45-50$

Servat E, Paturel E J, Lubes-Niel H, Kouame B, Masson J M, Travaglio M and Marieu B., 1999.Différents aspects de la variabilité de la pluviométrie en Afrique de l'Ouest et Centrale non sahélienne Rev. Sci. Eau, pp.12-26

Vissin E W., 2007. Impact de la variabilité climatique et de la dynamique des eaux de surface sur les écoulements du bassin béninois du fleuve Niger Thèse de doctorat de $3^{\circ}$ cycle, Université de Bourgogne, Dijon, 267p

Welcomme R L., 1971. Evaluation de la pêche intérieure au Dahomey, son état actuel et ses possibilités, Rôme: FAO, 95p.

Zannou A., 2011. Analyse et modélisation du Cycle Hydrologique Continental pour la Gestion Intégrée des Ressources en Eau au Bénin. Cas du Bassin de l'Ouémé à Bétérou Thèse de Doctorat, UAC, Bénin, $315 \mathrm{p}$. 